\title{
Statins and the risk of idiopathic venous thromboembolism
}

\author{
Chen-Chang Yang, ${ }^{1,2,3,4}$ Susan S. Jick $^{4}$ \& Hershel Jick ${ }^{4}$ \\ ${ }^{1}$ Department of Medicine, School of Medicine, National Yang-Ming University, Taipei, Taiwan, ${ }^{2}$ National Poison Center, Veterans General \\ Hospital-Taipei, Taipei, Taiwan 11217, ${ }^{3}$ Department of Epidemiology, Harvard School of Public Health, 677 Huntington Avenue, Boston, MA 02115 \\ and ${ }^{4}$ Boston Collaborative Drug Surveillance Program, Boston University School of Medicine, 11 Muzzey Street, Lexington, MA 02421, USA
}

\begin{abstract}
Aims To evaluate the association between current statin use and the risk of idiopathic venous thromboembolism (VTE).

Methods A population-based retrospective follow-up with a nested case-control analysis using the General Practice Research Database.

Results There were 72 cases of idiopathic VTE. Using normolipidaemic nonuse as the reference group, the adjusted relative risks for idiopathic VTE for current/recent statin use, past statin use, past other lipid-lowering drug use, and hyperlipidaemic nonuse were $0.8(0.3,2.7), 2.4(0.6,10.0), 1.8(0.4,7.4)$, and $0.9(0.4,2.0)$ in the follow-up analysis, and were $1.1(0.3,4.3), 3.7(0.6,24.1), 2.0(0.3,11.6)$, and $0.4(0.2,1.2)$ in the case-control analysis.

Conclusions Current statin use was not associated with a reduced risk of idiopathic VTE.
\end{abstract}

Keywords: HMG-CoA reductase inhibitor, idiopathic venous thromboembolism, lipid-lowering drug

\section{Introduction}

In clinical trials, 3-hydroxy-3-methylglutaryl coenzyme A (HMG-CoA) reductase inhibitors (statins) have been clearly shown to be effective in reducing the risk of cardiovascular diseases in hyperlipidaemic patients [1]. Recently, in the Heart and Estrogen/progestin Replacement Therapy (HERS) study, which was primarily designed for evaluating the relation between hormone replacement therapy and recurrent cardiovascular events [2], Grady et al. found that, in addition to an association between hormone therapy and increased risk of venous thromboembolism (VTE), the use of statins was related to a reduced risk of VTE (relative hazard 0.5, 95\% CI 0.2, 0.9). The authors postulated that the favourable mechanisms for statins on arterial thrombus formation might also decrease the risk of VTE.

Although this finding was somewhat unanticipated, such an effect may have important implications in public health since VTE, including deep vein thrombosis and

Correspondence: Dr Chen-Chang Yang, Boston Collaborative Drug Surveillance Program, Boston University School of Medicine, II Muzzey Street, Lexington, MA 0242I, USA.

Received I4 May 200I, accepted 30 August 2001. pulmonary embolism, is a potentially fatal disease and statins are widely used in clinical practice. To provide further information, we studied the association between exposure to statins and idiopathic VTE in the General Practice Research Database (GPRD).

\section{Methods}

The GPRD has been described elsewhere [3, 4]. Briefly, the GPRD contains computerized records for some 3 million residents in the UK enrolled with selected general practitioners (GPs), who use office computers to record anonymous information on patient demographics, diagnoses, referrals, hospitalizations, and drug prescriptions. The recorded information in the GPRD has been shown to be of high quality and is satisfactory for pharmacoepidemiologic studies [3, 4].

Within the GPRD, we selected three groups of people who were aged 40-79 years during January 1, 1991 through December 31, 1999. Group 1 comprised patients who received $\geq 1$ prescription for a lipid-lowering drug (LLD, including statin, fibrate, or other LLD). Group 2 included patients with a hyperlipidaemia diagnosis who did not receive LLDs. Group 3 was a random sample of 50000 patients who had neither a prescription for a LLD 
nor a diagnosis of hyperlipidaemia. For all subjects, we started the follow-up from January 1, 1991 or the date at which the prescription records began (whichever came later) until he/she developed VTE or any exclusion criteria, left the practice, died, became $\geq 80$ years of age, or the study period ended (whichever came first). All subjects with a history of VTE, cerebrovascular diseases, coronary or peripheral arterial diseases, heart failure, cancer (excluding nonmelanoma skin cancer), coagulopathies, vasculitis, chronic renal diseases, complicated hypertension or diabetes, alcohol/drug abuse, or epilepsy before the start of follow-up were excluded from the study base. We excluded these diseases because they are associated with vasculopathies, coagulation disorders, or immobilization, which are likely to be important medical risk factors for VTE. We also excluded patients $\geq 80$ years of age because there were few LLD exposures among these patients and they are usually more debilitated and may have unrecorded medical risk factors for VTE.

From the study base, we identified 352 subjects who were hospitalized with a first-time diagnosis of VTE. The date of hospitalization was defined as the index date. Two investigators reviewed their computerized information without knowledge of exposure. A case was considered as 'idiopathic' VTE if he/she was free of all aforementioned diseases and did not have major surgery, leg fracture, trauma, hospitalization, or pregnancy within 3 months before the index date. After record review, 126 possible cases remained.

We requested a copy of manual medical records from the GPs to validate the 126 potential cases. A total of 92 records were available for review. Final case status was classified as 'confirmed'; those with incident idiopathic VTE who needed hospitalization and anticoagulation, and whose manual records contained supporting objective evidence for VTE (e.g. positive venogram, $n=37$ ), or 'probable' idiopathic VTE; similar to confirmed cases, except that the diagnostic tests gave equivocal results or were absent from the discharge letter $(n=13)$. We also included 22 patients as probable cases whose manual records were not available for review, but who had a clearly documented anticoagulant-supported diagnosis of idiopathic VTE because in the review process, it was determined that $90.6 \%(48 / 53)$ of such patients who had available discharge letters were final cases.

We matched six controls to each case on age ( \pm 2 years), sex, practice, calendar time (controls were analysed at the same index date as their matched cases), and years of history in the GPRD ( \pm 1 years). The same exclusion criteria for cases were applied to all controls. All cases and controls had $\geq 1$ years of information in the GPRD before the index date.

Exposures were defined as follows: statins, other LLDs, mixed use ( $\geq 2$ LLDs concomitantly), hyperlipidaemic and normolipidaemic nonuse. For LLD exposures, we further divided the exposed person-time into current, recent, or past use. Because a prescription for a LLD usually lasts for 30 days and patients are not likely to be fully compliant, we defined current use as starting from the first day of the LLD prescription (after January 1, 1991) plus 45 days or until the first of the following occurred: use of the LLD was stopped; LLD in a different class was prescribed; or the follow-up ended. Recent use spanned from day 46-90; and past use started on day 91. All remaining person-time was counted as either hyperlipidaemic or normolipidaemic nonuse person-time. In the case-control analysis, current use was defined as having had the last prescription of a LLD $\leq 45$ days before the index date; recent use: 46-90 days; and past use: $\geq 91$ days.

We also evaluated the potential confounding effects of age (40-59 vs 60-79 years), calendar year (1991-95 vs 1996-99), and gender in the follow-up analysis; and smoking status (none, current, past, or unknown), body mass index (BMI, <25, 25-29.9, $\geq 30 \mathrm{~kg} \mathrm{~m}^{-2}$, unknown), and oestrogen use (none, current, past) in the case-control analysis. Subjects were defined as current oestrogen use if they received any oral contraceptives or hormone replacement therapy $\leq 6$ months before the index date.

\section{Statistical analysis}

Using normolipidaemic nonuse as the reference group, we employed Poisson regression to calculate crude and adjusted incidence rate ratios (IRRs) for idiopathic VTE in the follow-up analysis, while conditional logistic regression was used to estimate the unadjusted and adjusted odds ratios in the case-control analysis.

\section{Results}

The study base comprised 84093 individuals, including 22993 subjects who received $\geq 1$ prescription for LLDs during the study period (group 1), 11100 hyperlipidaemic patients who did not receive LLDs (group 2), and 50000 randomly selected normolipidaemic subjects (group 3). During the study period, 72 patients ( 37 confirmed and 35 probable) had an incident idiopathic VTE.

The total follow-up was 393176 person-years. The adjusted IRRs for current/recent statin use, past statin use, past other LLD use, and hyperlipidaemic nonuse were 0.8 $(0.3,2.7), 2.4(0.6,10.0), 1.8(0.4,7.4)$, and $0.9(0.4,2.0)$, compared with normolipidaemic nonuse. Because there were no cases in recent statin use, current/recent other LLD use, or all mixed use, and little information could be derived from these exposures, we combined certain exposures. The crude and adjusted IRRs for idiopathic VTE among various exposures are shown in Table 1. 
Table 1 Crude and adjusted incidence rate ratios by various exposures.

\begin{tabular}{|c|c|c|c|c|c|}
\hline Exposure & $\begin{array}{c}\text { Number of } \\
\text { cases }\end{array}$ & $\begin{array}{c}\text { Person-years of } \\
\text { exposure }\end{array}$ & $\begin{array}{c}\text { Crude incidence rate } \\
\text { per } 100000 \\
\text { person-years }\end{array}$ & $\begin{array}{c}\text { Crude incidence } \\
\text { rate } \\
\text { ratio }(95 \% \mathrm{CI})\end{array}$ & $\begin{array}{c}\text { Adjusted incidence } \\
\text { rate } \\
\text { ratio }(95 \% \mathrm{CI}) *\end{array}$ \\
\hline Normolipidaemic nonuse & 59 & 324376 & 18.2 & Reference & Reference \\
\hline Current/recent statin use & 3 & 14184 & 21.2 & $1.2(0.2,3.6)$ & $0.8(0.3,2.7)$ \\
\hline Past statin use & 2 & 3481 & 57.5 & $3.2(0.4,11.9)$ & $2.4(0.6,10.0)$ \\
\hline Current/recent other lipid-lowering drug use & 0 & 12563 & 0 & $0(0,1.7)$ & - \\
\hline Past other lipid-lowering drug use & 2 & 5099 & 39.2 & $2.2(0.3,8.1)$ & $1.8(0.4,7.4)$ \\
\hline Mixed use & 0 & 912 & 0 & $0(0,23.0)$ & - \\
\hline Hyperlipidaemic nonuse & 6 & 32561 & 18.4 & $1.0(0.4,2.3)$ & $0.9(0.4,2.0)$ \\
\hline
\end{tabular}

$\star$ Adjusted for age, calendar year, and sex.

In the adjusted analysis, older age, but not sex, was associated with the risk of idiopathic VTE (IRR 1.7, 95\% CI 1.0, 2.7).

A total of 432 controls, including 355 controls from the same practice as their cases and 77 controls from other practices, were matched to the 72 cases. The average years of follow-up and the mean age were similar between cases and controls. Cases were more likely than the controls to be current smokers and current oestrogen users. The distribution of patient characteristics and various exposures is shown in Table 2 .

The results of the matched analyses are also shown in Table 2. In these analyses, we included all cases and controls because the results were similar to those using either only confirmed cases or only controls matched to the cases on practice. Using normolipidaemic nonuse as the reference group, the ORs after adjusting for smoking, BMI, and oestrogen use were $1.1(0.3,4.3), 3.7$ (0.6, 24.1), 2.0 (0.3, 11.6), and $0.4(0.2,1.2)$, for current/recent statin use, past statin use, past other LLD use, and hyperlipidaemic nonuse. Current smoking, BMI $\geq 30 \mathrm{~kg} \mathrm{~m}^{-2}$, and current oestrogen use were all independently associated with the risk of idiopathic VTE. There was no effect modification by sex.

\section{Discussion}

The results from both the follow-up and the case-control analyses do not suggest that current statin use is associated with a reduced risk of idiopathic VTE. Both analyses also failed to show any association between the risk of idiopathic VTE and the use of other LLDs or hyperlipidaemia. However, these data do show a relation between the risk of idiopathic VTE and age (follow-up analysis); current oestrogen use, BMI $\geq 30.0 \mathrm{~kg} \mathrm{~m}^{-2}$ and current smoking (case-control analysis), the findings of which are consistent with previous studies on idiopathic VTE [5-12].

The results for current statin use from this study are different from the conclusion in the HERS trial [2], possibly because the study population and the definition of idiopathic VTE are not the same in the current study as in the HERS trial. In the HERS trial, the study population comprised patients with coronary or other atherosclerotic arterial diseases, while we excluded such people since we considered them at increased risk of VTE regardless of the exposure.

Studies on idiopathic VTE usually exclude cases with pre-existing coronary or peripheral arterial diseases because such patients are likely to be less healthy, and may be less mobile due to decreased cardiac output, peripheral oedema, or pain. Although there is no substantial evidence that atherosclerotic disease is a risk factor for VTE, both diseases share certain common risk factors, such as smoking, ageing, and obesity [13, 14]. With similar pathogenesis, it is possible that patients with atherosclerotic disease may be at increased risk of VTE. In an autopsy study of 337 adults, it was found that pulmonary artery atherosclerosis is associated with atherosclerosis of systemic arteries [15]. Moreover, the incidence of 'idiopathic' VTE in the placebo group in the HERS trial (23/10000 person-years) was substantially higher than the previously reported incidence of idiopathic VTE among women receiving hormone replacement therapy [6, 10], and was closer to the incidence of all VTE events [8]. Hence, in order to isolate the drug effect, VTE cases with preexisting atherosclerotic disease should not be considered as 'idiopathic'.

One of the major concerns in studying the association between statins and idiopathic VTE is the possibility that the patient characteristics, which are predictors of VTE, may be different between patients who received statins and users of other LLDs or nonusers (e.g. patients receiving LLDs for secondary prevention would have a higher risk of VTE than untreated hyperlipidaemic patients). In this study, by restricting our analysis to reasonably healthy subjects without prior history of VTE or other medical risk factors for VTE, we avoided the potential selection bias that could be caused by comorbidities with different risks of VTE between various exposure groups (e.g. LLD users $v s$ nonusers). We also matched cases and controls on 
Table 2 Distribution of patient characteristics and lipid-lowering drug exposure among all case and control patients and their association with VTE risk in multivariate analysis.

\begin{tabular}{|c|c|c|c|c|}
\hline & Case $(\mathrm{n}=72)$ & Control $(\mathrm{n}=432)^{\star}$ & Unadjusted odds ratio (95\% CI) & Adjusted odds ratio $(95 \% \mathrm{CI})$ \\
\hline \multicolumn{5}{|l|}{ Age (years) } \\
\hline $40-49$ & $10(13.9 \%)$ & $56(13.0 \%)$ & - & - \\
\hline $50-59$ & $23(31.9 \%)$ & $140(32.4 \%)$ & - & - \\
\hline $60-69$ & $23(31.9 \%)$ & $150(34.7 \%)$ & - & - \\
\hline $70-79$ & $16(22.2 \%)$ & $86(19.9 \%)$ & - & - \\
\hline \multicolumn{5}{|l|}{ Sex } \\
\hline Male & $36(50.0 \%)$ & $216(50.0 \%)$ & - & - \\
\hline Female & $36(50.0 \%)$ & $216(50.0 \%)$ & - & - \\
\hline \multicolumn{5}{|l|}{ Smoking } \\
\hline Nonsmoker & $41(56.9 \%)$ & $221(51.2 \%)$ & - & - \\
\hline Current & $17(23.6 \%)$ & $71(16.4 \%)$ & $1.4(0.7,2.7)$ & $2.0(1.0,4.4)$ \\
\hline Ex-smoker & $6(8.3 \%)$ & $70(16.2 \%)$ & $0.4(0.2,1.1)$ & $0.4(0.2,1.1)$ \\
\hline Unknown & $8(11.1 \%)$ & $70(16.2 \%)$ & $0.6(0.2,1.3)$ & $1.2(0.4,3.8)$ \\
\hline \multicolumn{5}{|l|}{ Body mass index $\left(\mathrm{kg} / \mathrm{m}^{2}\right)$} \\
\hline$<25.0$ & 19 (26.4\%) & $123(28.5 \%)$ & - & - \\
\hline $25.0-29.9$ & $25(34.7 \%)$ & $130(30.1 \%)$ & $1.2(0.6,2.3)$ & $1.9(0.9,3.7)$ \\
\hline$\geq 30.0$ & $17(23.6 \%)$ & $65(15.1 \%)$ & $1.7(0.8,3.6)$ & $2.5(1.1,5.7)$ \\
\hline Unknown & $11(15.3 \%)$ & $114(26.4 \%)$ & $0.6(0.3,1.3)$ & $0.6(0.2,1.7)$ \\
\hline \multicolumn{5}{|l|}{ Oestrogen use } \\
\hline None & $52(72.2 \%)$ & $366(84.7 \%)$ & - & - \\
\hline Current & $15(20.8 \%)$ & $40(9.3 \%)$ & $5.3(2.1,13.6)$ & $6.5(2.4,17.9)$ \\
\hline Past & $5(6.9 \%)$ & $26(6.0 \%)$ & $2.3(0.7,7.7)$ & $2.3(0.7,7.7)$ \\
\hline \multicolumn{5}{|l|}{ Exposures of interest } \\
\hline Normolipidaemic nonuse & $59(81.9 \%)$ & $329(76.2 \%)$ & - & - \\
\hline Current/recent statin use & $3(4.2 \%)$ & $18(4.2 \%)$ & $0.9(0.3,3.4)$ & $1.1(0.3,4.3)$ \\
\hline Past statin use & $2(2.8 \%)$ & $5(1.2 \%)$ & $2.0(0.3,11.3)$ & $3.7(0.6,24.1)$ \\
\hline $\begin{array}{l}\text { Current/recent other } \\
\text { lipid-lowering drug use }\end{array}$ & $0(0.0 \%)$ & $12(2.8 \%)$ & - & - \\
\hline $\begin{array}{l}\text { Past other lipid-lowering } \\
\text { drug use }\end{array}$ & $2(2.8 \%)$ & $6(1.4 \%)$ & $1.8(0.3,9.5)$ & $2.0(0.3,11.6)$ \\
\hline Mixed use & $0(0.0 \%)$ & $6(1.4 \%)$ & - & - \\
\hline Hyperlipidaemic nonuse & $6(8.3 \%)$ & $56(13.8 \%)$ & $0.6(0.2,1.5)$ & $0.4(0.2,1.2)$ \\
\hline
\end{tabular}

^Including 355 controls from the same general practice, and 77 controls selected from the other practices.

several covariates that might confound the association between LLDs and idiopathic VTE, and adjusted for other confounders in the analysis. Although incomplete control of BMI and smoking due to missing data could have resulted in residual confounding, controlling for them or excluding subjects with missing information in the multivariate analysis only slightly changed the ORs. Therefore, the magnitude of residual confounding, if present, is likely to be small.

The use of computerized prescription records to assess drug exposures is not subject to recall bias/error. Moreover, it has been shown that there is high concordance between the prescriptions recorded in the GPRD and the Prescription Pricing Authority reports [16]. The fact that most LLDs are prescribed on an outpatient basis and are generally filled continually, since hyperlipidaemia is a chronic disease, further lowered the potential for exposure misclassification. Further, case identification/ascertainment was virtually complete, and the process was not biased by drug exposure. All identified cases were carefully reviewed and we were able to validate most of the diagnoses. Therefore, cases were also unlikely to be misclassified.

In conclusion, although we can not completely rule out the possibility that statins may preferentially protect patients with pre-existing cardiovascular diseases from developing VTE, our results do not support the hypothesis that current statin use can reduce the risk of VTE in otherwise healthy subjects.

We thank the participating general practitioners for their excellent cooperation and generous help. The Boston Collaborative Drug Surveillance Program is supported in part by grants from AstraZeneca, Berlex Laboratories, Boehringer Ingelheim Pharmaceuticals, Boots Healthcare International, Bristol-Myers Squibb Pharmaceutical Research Institute, GlaxoSmithKline, Hoffmann-La Roche, Janssen Research Foundation and Novartis Farmacéutica. 


\section{References}

1 LaRosa JC, He J, Vupputuri S. Effects of statins on risk of coronary heart disease: a meta-analysis of randomized controlled trials. JAMA 1999; 282: 2340-2346.

2 Grady D, Wenger NK, Herrington D, et al. Postmenopausal hormone therapy increases risk for venous thromboembolic disease. the Heart and Estrogen/progestin replacement Study. Ann Int Med 2000; 132: 689-696.

3 Jick J, Jick SS, Derby LE. Validation of information recorded on general practitioner based computerised data resource in the United Kingdom. Br Med J 1991; 302: 766-768.

4 Jick H, Terris BZ, Derby LE, Jick SS. Further validation of information recorded on a general practitioner based computerized data resource in the United Kingdom. Pharmacoepidemiol Drug Saf 1992; 1: 347-349.

5 Jick H, Jick SS, Guerwich V, Myers MW, Vasilakis C. Risk of idiopathic cardiovascular death and nonfatal venous thromboembolism in women using oral contraceptives with differing progestagen components. Lancet 1995; 346: 1589-1593.

6 Pérez-Gutthann S. Garcia Rodríguez LA, Castellsague J, Oliart AD. Hormone replacement therapy and risk of venous thromboembolism: population based case-control study. Br Med J 1997; 314: 796-800.

7 Jick H, Kaye JA, Vasilakis-Scaramozza C, Jick SS. Risk of venous thromboembolism among users of third generation of oral contraceptives compared with users of oral contraceptives with levonorgestrel before and after 1995: cohort and case-control analysis. Br Med J 2000; 321: 1190-1195.

8 Anderson FA, Wheeler B, Goldberg RJ, et al. A population-based perspective of the hospital incidence and case-fatality rates of deep vein thrombosis and pulmonary embolism. Arch Int Med 1991; 151: 933-938.

9 World Health Organization Collaborative Study of Cardiovascular Disease and Steroid Hormone Contraception. Venous thromboembolic disease and combined oral contraceptives: results of international multicenter case-control study. Lancet 1995; 346: 1575-1582.

10 Daly E, Vessey MP, Hawkins MM, Carson JL, Gough P, Marsh S. Risk of venous thromboembolism in users of hormone replacement therapy. Lancet 1996; 348: 977-980.

11 Meier CR, Jick H. Tamoxifen and risk of idiopathic venous thromboembolism. Br J Clin Pharmacol 1998; 45: $608-612$.

12 Vasilakis C, Jick SS, Jick H. The risk of venous thromboembolism in users of postcoital contraceptive pills. Contraception 1999; 59: 79-83.

13 Fruzzetti F. Hemostatic effects of smoking and oral contraceptive use. Am J Obstet Gynecol 1999; 180: S369-S374.

14 Expert Panel on Detection, Evaluation, Treatment of High Blood Cholesterol in Adults. Second report of the National Cholesterol Education Program (NCEP) Expert Panel on Detection, Evaluation, and Treatment of High Blood Cholesterol in Adults (Adult Treatment Panel II). Circulation 1994; 89: 1333-1445.

15 Moore GW, Smith RR, Hutchins GM. Pulmonary artery atherosclerosis. correlation with systemic atherosclerosis and hypertensive pulmonary vascular disease. Arch Pathol Laboratory Med 1982; 106: 378-380.

16 Garcia Rodríguez LA, Pérez-Gutthann S, Jick SS. The UK General Practice Research Database. In Pharmacoepidemiology, 3rd edn, ed. Strom, BL. New York: John Wiley \& Sons Ltd, 2000: $375-385$. 